\title{
Microstructures in Newly-Realized LnMN3 Phases
}

Allison Mis ${ }^{1}$, Rachel Sherbondy ${ }^{1}$, Andriy Zakutayev ${ }^{2}$ and Geoff Brennecka ${ }^{1}$

${ }^{1}$ Colorado School of Mines, United States, ${ }^{2}$ National Renewable Energy Laboratory, United States

Perovskite materials have long been studied for their abundance and applicability to a wide variety of materials problems. Much of this research has been focused on oxide and halide perovskites, but nitride perovskites have been predicted to be stable, particularly those that take the form $L n M N_{3}$, where $L n=L a$, $\mathrm{Ce}, \mathrm{Eu}, \mathrm{Yb}$, and $\mathrm{M}=\mathrm{W}$, Re. Recent reports [1] prove that $\mathrm{LaWN}_{3}$ can be grown as a perovskite with minimal oxygen contamination. This novel material's properties, including notable piezoelectric response, provide motivation for further materials discovery efforts in this area.

Various lanthanide-metal pairings were studied, and several show promise to expand the number of known nitride perovskite materials. Thin-film samples were grown and characterized using the National Renewable Energy Laboratory's suite of combinatorial RF sputter growth tools and various property mapping capabilities. Each growth produced a film with a range of compositions that included the target stoichiometry. X-ray diffraction patterns are consistent with perovskite or fluorite structures, however it is not definitive from XRD which structure is present.

In this work, we present a microscopy investigation of these films complementary to the above highthroughput characterization. Multiple compositions of $(\mathrm{Ce}, \mathrm{Mo}) \mathrm{N}$ and $(\mathrm{Ce}, \mathrm{W}) \mathrm{N}$ were studied. Characterization of these films includes chemical mapping using energy-dispersive $\mathrm{X}$-ray spectroscopy (EDS), selected-area electron diffraction, and a study of lattice spacings using high-resolution transmission electron microscopy (TEM). This work clarifies whether these new films are indeed perovskites and yields some surprising information about film structure, an example of which is shown below.

Initial transmission electron microscopy TEM imaging of a $(\mathrm{Ce}, \mathrm{Mo}) \mathrm{N}$ sample, shown in figure 1 , shows multiple layers in the plane of the film. Elemental mapping of these layers using EDS reveals cation segregation, as shown by the variation in $\mathrm{Ce}$ and Mo. There is also oxygen contamination, the severity of which is correlated with the amount of $\mathrm{Ce}$ : Ce-rich layers show a higher proportion of oxygen. This work presents an in-depth look at the structural properties of some of the newest lanthanide-metalnitrides.
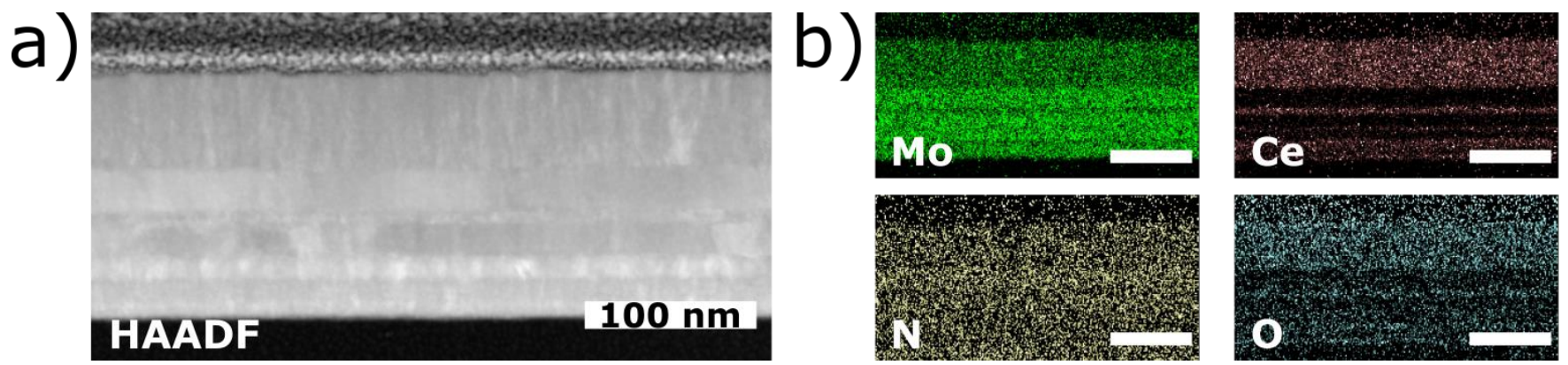

Figure 1. Fig. 1: a) cross-sectional HAADF imaging of a (Ce,Mo)N film showing in-plane layers in the film. b) EDS mapping of the (Ce,Mo)N film shows cation segregation and correlated oxygen contamination 


\section{References}

[1] Talley, et al. 2020. "Synthesis of Ferroelectric LaWN3 - the First Nitride Perovskite." arXiv:2001.00633 [cond-mat.mtrl-sci] 